\title{
Can We Accomplish Better Oncological Results with Robot-Assisted Radical Prostatectomy?
}

\author{
Ali Riza Kural, MD, Can Obek, MD, FEBU, and Tunkut Doganca, MD, FEBU ${ }^{2}$
}

\begin{abstract}
Surgical removal with radical prostatectomy has been a cornerstone for the treatment of prostate cancer and is associated with level 1 evidence for survival advantage compared with watchful waiting. Since the first structured robotic program was launched in 2000, robot-assisted radical prostatectomy (RARP) has had a rapid diffusion and surpassed its open radical prostatectomy (ORP) and laparoscopic radical prostatectomy (LRP) counterparts in the United States and is progressively expanding in other countries. Interestingly, this common acceptance of RARP was initially driven in the paucity of robust clinical evidence. There is still lack of level 1 evidence with prospective randomized trials on the oncologic outcomes of RARP. In that scenario, the clinician has to rely on retrospective data and systemic and meta-analyses. In comparison with ORP and LRP, RARP has proven to reach at least equivalent oncological outcomes. Lower rate of positive surgical margins may probably be achieved with RARP in pT2 patients. Although urologists were initially reluctant to embrace RARP in highrisk patients and lymph node yield was low, contemporary series have revealed that RARP and extended lymphadenectomy may be safely performed with obtaining similar (or better) nodal yields compared with ORP. Surgeon experience is universally of utmost importance in obtaining good outcomes. We will need to wait for long-term results of contemporary series to comprehend the impact of RARP on cancer-specific survival and overall survival. Using novel imaging before surgery and frozen section analysis during surgery may allow for superior oncological outcomes.
\end{abstract}

Keywords: prostate cancer, robotics, robot-assisted radical prostatectomy

\section{Introduction}

$\mathbf{R}$ ADICAL PROSTATECTOMY HAS been a cornerstone for the treatment of prostate cancer ( $\mathrm{PCa})$ and is associated with level 1 evidence for survival advantage compared with watchful waiting. ${ }^{1}$ Patients undergoing open radical prostatectomy (ORP) experience 15-year cancer-specific survival (CSS) rates between $80 \%$ and $93 \%$ and biochemical recurrence (BCR) rates as low as $25 \% .^{2}$

Along with the advances in technology, robot-assisted radical prostatectomy (RARP), using the da Vinci platform (Intuitive Surgical, Inc., Sunnyvale, CA), was launched as a novel procedure. Robotic technology has well-known technical advantages that could potentially improve the quality of surgery. Three-dimensional view of surgical field, better illumination, higher magnification, greater and more intuitive movement capability of the robotic arms, minimization of tremor, and venous tamponade of pneumoperitoneum are the typical benefits that may translate into better oncologic outcomes for PCa surgery.
Since the first structured program in 2000, RARP has had rapid diffusion and surpassed its open and laparoscopic counterparts in the United States and is progressively expanding in other countries. ${ }^{3}$

Interestingly, this common acceptance of RARP was initially driven in the paucity of robust clinical evidence. Factors such as patient-directed marketing and interhospital competition to offer the state-of-the-art technology acted as the main driving factors. ${ }^{4}$ Nearly two decades after its initial description, we still lack level 1 evidence about its impact on the oncologic outcomes. There is abundance of data that blood loss is reduced and length of hospital stay and convalescence is shortened compared with ORP. ${ }^{5}$

\section{Oncologic Outcomes}

Data on oncologic outcomes are limited, scarce, and difficult to interpret. There is no large-scale randomized trial comparing RARP head-to-head with open or standard laparoscopic surgery. The only prospective randomized trial

${ }^{1}$ Department of Urology, Acibadem University, Istanbul, Turkey.

${ }^{2}$ Department of Urology, Acibadem Taksim Hospital, Istanbul, Turkey. 
comparing RARP and ORP has recently been published with only 12 weeks of follow-up. ${ }^{5}$ The only oncologic clue (with such short follow-up) from this study is that positive surgical margin (PSM) rates were similar. Most evidence comes from observational cohort studies and meta-analyses. ${ }^{4}$ These studies compare noncontemporary series with retrospective data and short-term follow-up. Cohorts are heterogeneous and adjustments for confounding factors may be suboptimal. The most important parameters, CSS and overall survival, are not reported since they require many years of follow-up, specifically in a disease with a long natural history such as PCa. Although not the ideal surrogate for oncologic outcomes, literature is typically limited to the rate of PSM, requirement for additional therapy, and incidence of BCR.

\section{Reviews, meta-analysis, and comparative series}

Novara and colleagues published a systematic review and meta-analysis of studies reporting oncologic outcomes after RARP. ${ }^{6}$ The prevalence of PSM ranged from $6.5 \%$ to $32 \%$ (mean 15\%). Fellowship training was associated with lower rates. There was no difference between the transperitoneal $v s$ extraperitoneal approach or preservation of bladder neck. The technique used to control the dorsal venous complex had an impact on margins. Authors mentioned two series published before 2012 regarding BCR. In the larger, Vattikuti Institute reported BCR-free rates of $90 \%, 87 \%$, and $81 \%$ at 3 , 5 , and 7 years, respectively. The conclusion was that RARP achieves similar BCR rates to ORP when performed by experienced surgeons. ${ }^{6}$

Moran and colleagues published a review article comparing RARP, ORP, and standard laparoscopic radical prostatectomy (LRP) in $2013 .{ }^{7}$ Of the 51 articles analyzed, only 3 were rated as high and 14 as good quality, demonstrating the inferior quality of publications on this topic. When analyzed for PSM rates, outcome for pT2 patients was superior with RARP compared with ORP (RR 0.63, 95\% CI 0.49, 0.81, $p<0.001)$. Moreover, lower rate of PSM was obtained together with improved urinary and sexual function at 12 months. However, there was no difference for pT3 cases. As for RARP vs LRP, PSM rates were equivalent. Of note, when analysis was restricted to studies of higher methodological quality (6 studies with 1130 patients), significant difference for PSM between open and robotic surgery diminished. PSM is associated with a twofold increased hazard of biochemical relapse; however, its association with more robust clinical endpoints remains controversial. ${ }^{8}$

Tewari and colleagues reported on a systematic review and meta-analysis of ORP, RARP, and LRP. ${ }^{8}$ They used propensity score matching to adjust for different variables. Overall and pT2 PSM rates were similar in ORP and RARP; whereas RARP was significantly superior to LRP in both comparisons.

In a more recent systematic review of the comparison of the three methods, De Carlo and colleagues reported that RARP achieved significantly lower rates of PSM for pT2 patients compared with both the open and laparoscopic counterparts. ${ }^{9}$

On the 10-year follow-up results reported from Vattikuti Institute, actuarial BCR-free, metastasis-free, and CS survival rates were $73.1 \%, 97.5 \%$, and $98.8 \%$, respectively. ${ }^{10}$ Authors concluded that RARP confers effective long-term cancer control.
Ploussard and colleagues compared RARP vs pure extraperitoneal LRP and observed the superiority of RARP for obtaining safer margins in pT2 disease. ${ }^{11}$ Once again, this difference disappeared for pT3 cancer. BCR was similar and surgeon volume did not have an impact. This contradicts with other data depicting higher rates of PSM with lower case numbers. ${ }^{11}$

In a contemporary, large national cohort study from the United States using the National Cancer Database on 96,935 patients, RARP was independently associated with clinically meaningful reductions in PSMs, postoperative radiation therapy, and 30-day mortality rate compared with ORP. ${ }^{12}$ The oncologic benefit was primarily in patients with organconfined (pT2) cancer. As the authors stated, this would suggest that tumor biology rather than surgical approach dictates margin status when extraprostatic extension is present.

\section{Data on high-risk patients}

New concepts of multimodal therapy have made surgery an option in high-risk (HR) nonmetastatic patients. ${ }^{13}$ Although associated with increased incidence of BCR and secondary therapy, HR patients have 10-year CSS estimates in the range of $90 \%$ following ORP. ${ }^{14}$ Interestingly, the preference toward surgical treatment in HR disease has overlapped with the RARP era. Given the relative novelty of RARP, there was initial reluctance to embrace RARP for this subset of patients. Some of the potential reasons of discouragement for surgery in HR disease included inexperience with RARP in that scenario, lack of haptic feedback, and potential difficulty of performing extended (e) lymph node dissection (LND) with the robot. ${ }^{15}$

Silberstein and colleagues published their experience in a case-mix-adjusted comparison of early oncological outcomes of ORP and RARP performed by experienced surgeons. ${ }^{16}$ Importantly, the cohort contained a majority of patients with intermediate and HR features. All surgeons performed complete pelvic LND. They found no evidence to suggest that RARP results in inferior oncological outcomes with respect to PSM and BCR-free survival, even for patients with HR cancer. Differences between surgeons were larger than differences between surgical approaches. They concluded that when performed optimally, RARP may be safely performed in HR disease.

Abdollah and colleagues recently reported on long-term cancer control outcomes in HR cases with RARP. ${ }^{15}$ They evaluated 1100 patients; at 10 years, BCR-free, clinical recurrence-free, and salvage therapy rates were $50 \%, 87 \%$, and $37 \%$, respectively. They concluded that these results are in alignment with previously reported results for ORP. Assessing the outcomes of ORP and RARP in a small cohort of $\mathrm{HR}$ men at a single institution, Punnen and colleagues reported comparable PSM and BCR rates. ${ }^{17}$ In a relatively new singlesurgeon series, Lee and colleagues assessed the oncologic outcomes in ORP vs RARP in HR patients. ${ }^{18}$ After adjustment of preoperative variables, 5-year BCR-free rates were similar (48.5\% for ORP and 59.6\% for RARP, $p=0.13$ ). Surgical approach did not predict BCR in multivariate analysis.

\section{$R A R P$ and $L N D$}

Lymphadenectomy at the time of RP has diagnostic, prognostic, and potentially therapeutic intent. It confers information 
about the level and extent of nodal involvement and may guide initiation of earlier adjuvant therapy. In a study randomizing men with HR disease to standard $v s$ extended dissection, BCRfree survival was improved at 6 years in the extended group $(51 \%$ vs $71 \%){ }^{14}$

As stated by Dr. Montorsi in an editorial, pelvic LND is not yet widely used, and this remains a significant limitation in the RARP arena. ${ }^{13}$ However, as we will try to elucidate in the following, this is not a problem of the robot, but of the surgeon. Initial experience with RARP was indeed associated with a lower likelihood of receiving an LND. ${ }^{17}$ Nevertheless, as urologists gain experience with utilizing the robot and operate on HR patients, data accumulate on the details of lymphadenectomy using the device.

The role of RARP and pelvic LND was evaluated in a systematic review, where data on 12 publications and 1360 patients were analyzed. ${ }^{14}$ Patients were risk stratified by either D'Amico or NCCN criteria. Nerve-sparing surgery rate was high at $73 \%$. eLND was consistently used in only 4 series (33\%) with a median node yield of 18 . Review showed that robotic eLND can safely achieve lymph node (LN) yields of 16 to 24. Probability of nodal disease detection increased with more extensive dissection. Secondary treatment was administered in $9 \%$ to $23 \%$ of patients at short-term followup in line with open series.

In our RARP experience, eLND is deliberately used. In HR patients, we have recently extended our dissection planes to the bifurcation of the aorta and include the presacral nodes in our template. For the most recent consecutive $25 \mathrm{HR}$ and very HR patients, the mean number of nodes removed was 34 (range 15-70) (unpublished data). This is clear evidence that even eLND may be safely performed during RARP.

\section{Suggestions with Potential for Improving Oncologic Outcomes}

\section{Using multiparametric MRI for surgical planning}

Data on the impact of using multiparametric prostate MRI for local setting and thus planning surgical planes (wide resection for pT3 vs NS for pT2) remain controversial. ${ }^{19,20} \mathrm{We}$ believe that multiparametric MRI is a good tool to demonstrate macroscopic invasion; however, it may not be precise enough to detect microscopic extracapsular extension.

\section{Frozen section analysis during surgery}

As stated above, preoperative multiparametric MRI cannot predict T3 disease consistently. The clinician considers multiple factors, including prostate-specific antigen level, prostate examination, clinical risk category, MRI findings, biopsy findings, baseline potency, and patient expectations, when planning for the details of surgical planes and nerve sparing. However, any urologist performing this surgery is cognizant of the fact that none of the abovementioned information guarantees either the optimum strategy for nerve sparing or obtaining negative surgical margins. One of the modifiable factors for achieving higher rates of negative surgical margins during surgery (open, laparoscopic, or RARP) would be to perform intraoperative frozen section analysis (FSA). This allows for further resection at the location, where the margin would have been positive without FSA, and thus achieving a significantly higher rate of oncologically safer margins.
A systematic approach of FSA was introduced by Schlomm and colleagues at Martini Clinic, where they submitted the entire neurovascular-adjacent prostatic tissue for intraoperative assessment by dedicated genitourinary pathologists (NeuroSAFE). ${ }^{21}$ They concluded that this technique was a useful adjunct to decrease the rate of PSMs and safe preservation of neurovascular bundles in HR patients undergoing open or RARP, who otherwise would have been considered candidates for non-nerve-sparing surgery. von Bodman and colleagues reported converting $92 \%$ of patients with intraoperative PSMs to negative margins. ${ }^{22}$ Our group has described a new technique for intraoperative FSA. ISTANBUL Preserve (Intra-Surgical Total and Re-Constructible Pathological Prostate Examination for Safer Margins and Nerve Preservation) allows for the entire margin surrounding the prostate to be examined. ${ }^{23}$ Thus, FSA is not limited to the area adjacent to the neurovascular bundle, but rather covers the whole gland. Another major advantage is that complete whole mount reconstruction of the prostate is possible with this novel technique with negligible tissue lost at FSA. In our experience with 170 patients, conversion of a PSM to negative was achieved in $85 \%$. By intraoperative navigation through FSA, the PSM rate decreased from $22.5 \%$ to $7.5 \%$. The approach in the presence of a PSM during surgery is individualized based on the extent and Gleason pattern at the PSM. No further tissue was excised at the presence of a few glands with Gleason pattern 3. In patients with extensive PSM with Gleason pattern 4 or 5 , the entire bundle was resected. For patients with small margin positivity with Gleason pattern 4, the bundle was partially resected. The surgeon also gains information about accurate Gleason grading during surgery (may differ from biopsy) and occasionally changes the extent of LND.

\section{Novel imaging techniques in primary lymph node staging for $\mathrm{HR} P C a$}

${ }^{68}$ Gallium-PSMA positron emission tomography (PET) scan is the most recent addition to the novel molecular imaging modalities for staging of $\mathrm{PCa}$, in which a small molecule ligand of PSMA is labeled with a positron-emitting radionuclide ${ }^{68}$ Gallium. ${ }^{24} \mathrm{We}$ believe that there is a potential to use this or similar advanced imaging modalities for precise lymphatic staging in the future. It would potentially allow the surgeon to modify the dissection template for the individual patient, obtaining better regional control of the disease. Two groups have published their results on the predictive value of PSMA PET/CT for LN metastases. ${ }^{25}$ Budäus and colleagues reported their experience on 30 patients with HR PCa from Martini Clinic Prostate Cancer Center, while Maurer and colleagues reported on results of 130 patients with intermediate to HR PCa from the University of Munich. ${ }^{25,26}$ The sensitivity and specificity values were $33.3 \%$ and $100 \%$ at Martini Clinic and $65.9 \%$ and $98.9 \%$ at the University of Munich. Herlemann and colleagues have recently demonstrated that PSMA scan before surgery identifies cancer outside the standard LND template, allowing for improved identification of LN-positive disease at the time of surgery. ${ }^{27}$ ${ }^{18}$ F-fluoro- $5 \alpha$-dihydrotestosterone, anti-1-amino-3- ${ }^{18}$-Ffluorocyclobutane-1-carboxylic acid, and ${ }^{11} \mathrm{C}$-acetate are other investigational PET CT modalities.

Ultrasensitive superparamagnetic iron oxide particles have recently been investigated at a few centers. ${ }^{28}$ Lymphotropic 
nanoparticle-enhanced MRI, also called magnetic resonance lymphography, is a technique with a reported sensitivity of $65 \%$ to $92 \%$ and specificity of $93 \%$ to $98 \%$ in detecting LN metastases and allows for detection of nonenlarged LNs. ${ }^{29}$ Further evidence will prove the utility of these novel imaging modalities in daily practice. ${ }^{30}$

\section{Conclusion}

There is a lack of level 1 evidence to answer the question whether better oncological results are obtained with RARP. Published data are limited, scarce, and difficult to interpret. However, growing evidence suggests that outcomes are at least equivalent to ORP and LRP in comparisons of proxies such as PSM, BCR-free rates, and need for additional treatment. The rates of PSMs are probably lower with RARP in pT2 patients. In HR disease, RARP and extended LND may be safely performed, obtaining similar (or better) LN yields compared with ORP. Molecular imaging may improve surgical planning and using FSA during surgery may allow for superior functional and oncological outcomes.

\section{Author Disclosure Statement}

No competing financial interests exist.

\section{References}

1. Bill-Axelson A, Holmberg L, Garmo H, Rider JR, Taari K, Busch C, et al. Radical prostatectomy or watchful waiting in early prostate cancer. N Engl J Med 2014;370:932-942.

2. Boorjian SA, Eastham JA, Graefen M, Guillonneau B, Karnes RJ, Moul JW, et al. A critical analysis of the longterm impact of radical prostatectomy on cancer control and function outcomes. Eur Urol 2012;61:664-675.

3. Menon M, Shrivastava A, Tewari A, Sarle R, Hemal A, Peabody JO, et al. Laparoscopic and robot assisted radical prostatectomy: Establishment of a structured program and preliminary analysis of outcomes. J Urol 2002;168:945949.

4. Leow JJ, Chang SL, Meyer CP, Wang Y, Hanske J, Sammon JD, et al. Robot-assisted versus open radical prostatectomy: A contemporary analysis of an all-payer discharge database. Eur Urol 2016;70:837-845.

5. Yaxley JW, Coughlin GD, Chambers SK, Occhipinti S, Samaratunga H, Zajdlewicz L, et al. Robot-assisted laparoscopic prostatectomy versus open radical retropubic prostatectomy: Early outcomes from a randomised controlled phase 3 study. Lancet 2016;388:1057-1066.

6. Novara G, Ficarra V, Mocellin S, Ahlering TE, Carroll PR, Graefen M, et al. Systematic review and meta-analysis of studies reporting oncologic outcome after robot-assisted radical prostatectomy. Eur Urol 2012;62:382-404.

7. Moran PS, O’Neill M, Teljeur C, Flattery M, Murphy LA, Smyth G, et al. Robot-assisted radical prostatectomy compared with open and laparoscopic approaches: A systematic review and meta-analysis. Int J Urol 2013;20:312321.

8. Tewari A, Sooriakumaran P, Bloch DA, Seshadri-Kreaden U, Hebert AE, Wiklund P. Positive surgical margin and perioperative complication rates of primary surgical treatments for prostate cancer: A systematic review and metaanalysis comparing retropubic, laparoscopic, and robotic prostatectomy. Eur Urol 2012;62:1-15.
9. De Carlo F, Celestino F, Verri C, Masedu F, Liberati E, Di Stasi SM. Retropubic, laparoscopic, and robot-assisted radical prostatectomy: Surgical, oncological, and functional outcomes: A systematic review. Urol Int 2014;93:373-383.

10. Diaz M, Peabody JO, Kapoor V, Sammon J, Rogers CG, Stricker H, et al. Oncologic outcomes at 10 years following robotic radical prostatectomy. Eur Urol 2015;67:11681176.

11. Ploussard G, de la Taille A, Moulin M, Vordos D, Hoznek A, Abbou CC, et al. Comparisons of the perioperative, functional, and oncologic outcomes after robot-assisted versus pure extraperitoneal laparoscopic radical prostatectomy. Eur Urol 2014;65:610-619.

12. Pearce SM, Pariser JJ, Karrison T, Patel SG, Eggener SE. Comparison of perioperative and early oncologic outcomes between open and robotic assisted laparoscopic prostatectomy in a contemporary population based cohort. J Urol 2016;196:76-81.

13. Montorsi F. Robotic prostatectomy for high-risk prostate cancer: Translating the evidence into lessons for clinical practice. Eur Urol 2014;65:928-930.

14. Yuh B, Artibani W, Heidenreich A, Kimm S, Menon M, Novara G, et al. The role of robot-assisted radical prostatectomy and pelvic lymph node dissection in the management of high-risk prostate cancer: A systematic review. Eur Urol 2014;65:918-927.

15. Abdollah F, Sood A, Sammon JD, Hsu L, Beyer B, Moschini M, et al. Long-term cancer control outcomes in patients with clinically high-risk prostate cancer treated with robot-assisted radical prostatectomy: Results from a multi-institutional study of 1100 patients. Eur Urol 2015; 68:497-505.

16. Silberstein JL, Su D, Glickman L, Kent M, Keren-Paz G, Vickers AJ, et al. A case-mix-adjusted comparison of early oncological outcomes of open and robotic prostatectomy performed by experienced high volume surgeons. BJU Int 2013;111:206-212.

17. Punnen S, Meng MV, Cooperberg MR, Greene KL, Cowan JE, Carroll PR. How does robot-assisted radical prostatectomy (RARP) compare with open surgery in men with high-risk prostate cancer? BJU Int 2013;112:E314-E320.

18. Lee D, Choi SK, Park J, Shim M, Kim A, Lee S, et al. Comparative analysis of oncologic outcomes for open vs. robot-assisted radical prostatectomy in high-risk prostate cancer. Korean J Urol 2015;56:572-579.

19. Lee H, Kim CK, Park BK, Sung HH, Han DH, Jeon HG, et al. Accuracy of preoperative multiparametric magnetic resonance imaging for prediction of unfavorable pathology in patients with localized prostate cancer undergoing radical prostatectomy. World J Urol 2016. [Epub ahead of print]; DOI: 10.1007/s00345-016-1948-6.

20. Bianchi R, Cozzi G, Petralia G, Alessi S, Renne G, Bottero $\mathrm{D}$, et al. Multiparametric magnetic resonance imaging and frozen-section analysis efficiently predict upgrading, upstaging, and extraprostatic extension in patients undergoing nerve-sparing robotic-assisted radical prostatectomy. Medicine (Baltimore) 2016;95:e4519.

21. Schlomm T, Tennstedt P, Huxhold C, Steuber T, Salomon G, Michl U, et al. Neurovascular structure-adjacent frozensection examination (NeuroSAFE) increases nerve-sparing frequency and reduces positive surgical margins in open and robot-assisted laparoscopic radical prostatectomy: Experience after 11,069 consecutive patients. Eur Urol 2012; 62:333-340. 
22. von Bodman C, Brock M, Roghmann F, Byers A, Loppenberg B, Braun $\mathrm{K}$, et al. Intraoperative frozen section of the prostate decreases positive margin rate while ensuring nerve sparing procedure during radical prostatectomy. J Urol 2013;190:515-520.

23. Saglican Y, Obek C, Tufek I, Tuna MB, Doganca T, Mourmouris $\mathrm{P}$, et al. Intra-Surgical Total and ReConstructible Pathological Prostate Examination for Safer Margins and Nerve Preservation: ISTANBUL Preserve, MP13-14. In: 34th World Congress of Endourology \& SWL, New York, United States; Mary Ann Liebert, Inc., 2016.

24. Afshar-Oromieh A, Avtzi E, Giesel FL, Holland-Letz T, Linhart HG, Eder M, et al. The diagnostic value of PET/CT imaging with the (68)Ga-labelled PSMA ligand HBED-CC in the diagnosis of recurrent prostate cancer. Eur J Nucl Med Mol Imaging 2015;42:197-209.

25. Maurer T, Gschwend JE, Rauscher I, Souvatzoglou M, Haller B, Weirich G, et al. Diagnostic efficacy of (68)gallium-PSMA positron emission tomography compared to conventional imaging for lymph node staging of 130 consecutive patients with intermediate to high risk prostate cancer. J Urol 2016;195:1436-1443.

26. Budäus L, Leyh-Bannurah SR, Salomon G, Michl U, Heinzer H, Huland $\mathrm{H}$, et al. Initial experience of (68)GaPSMA PET/CT imaging in high-risk prostate cancer patients prior to radical prostatectomy. Eur Urol 2016;69: 393-396.

27. Herlemann A, Wenter V, Kretschmer A, Thierfelder KM, Bartenstein P, Faber C, et al. 68Ga-PSMA positron emission tomography/computed tomography provides accurate staging of lymph node regions prior to lymph node dissection in patients with prostate cancer. Eur Urol 2016;70: 553-557.

28. Winter A, Woenkhaus J, Wawroschek F. A novel method for intraoperative sentinel lymph node detection in prostate cancer patients using superparamagnetic iron oxide nanoparticles and a handheld magnetometer: The initial clinical experience. Ann Surg Oncol 2014;21:4390-4396.
29. Fortuin AS, Smeenk RJ, Meijer HJ, Witjes AJ, Barentsz JO. Lymphotropic nanoparticle-enhanced MRI in prostate cancer: Value and therapeutic potential. Curr Urol Rep 2014; 15:389.

30. Sankineni S, Brown AM, Fascelli M, Law YM, Pinto PA, Choyke PL, et al. Lymph node staging in prostate cancer. Curr Urol Rep 2015;16:30.

Address correspondence to: Tunkut Doganca, MD, FEBU Department of Urology Acibadem Taksim Hospital Nizamiye Mah. Inonu Cad. No 1-9 Sisli Istanbul 34300 Turkey

E-mail: tunkutdoganca@gmail.com

$\begin{aligned} & \text { Abbreviations Used } \\ & \mathrm{BCR}=\text { biochemical recurrence } \\ & \mathrm{CSS}=\text { cancer-specific survival } \\ & \mathrm{eLND}=\text { extended lymph node dissection } \\ & \mathrm{FSA}=\text { frozen section analysis } \\ & \mathrm{HR}=\text { high-risk } \\ & \mathrm{LN}=\text { lymph node } \\ & \mathrm{LND}=\text { lymph node dissection } \\ & \mathrm{LRP}=\text { laparoscopic radical prostatectomy } \\ & \mathrm{MRI}=\text { magnetic resonance imaging } \\ & \mathrm{ORP}=\text { open radical prostatectomy } \\ & \mathrm{PCa}=\text { prostate cancer } \\ & \mathrm{PET} \mathrm{CT}=\text { positron emission tomography- } \\ & \mathrm{PSM}=\text { computed tomography } \\ & \mathrm{RARP}=\text { robotive surgical margin } \\ &\end{aligned}$

